(C) The Authors 2015. This is an Open Access article, distributed under the terms of the Creative Commons Attribution licence (http:// creativecommons.org/licenses/by/3.0/), which permits unrestricted re-use, distribution, and reproduction in any medium, provided the original work is properly cited.

\title{
Effects of a food-based intervention on markers of micronutrient status among Indian women of low socio-economic status
}

\author{
Sarah H. Kehoe ${ }^{1 *}$, Harsha Chopra ${ }^{2}$, Sirazul A. Sahariah ${ }^{2}$, Dattatray Bhat ${ }^{3}$, Renuka P. Munshi ${ }^{4}$, \\ Falguni Panchal ${ }^{4}$, Stephen Young ${ }^{5}$, Nick Brown ${ }^{1}$, Dnyaneshwar Tarwande ${ }^{6}$, Meera Gandhi $^{2}$, \\ Barrie M. Margetts ${ }^{7}$, Ramesh D. Potdar ${ }^{2}$ and Caroline H. D. Fall ${ }^{1}$ \\ ${ }^{1}$ Medical Research Council Lifecourse Epidemiology Unit, University of Southampton, Southampton, UK \\ ${ }^{2}$ Centre for Study of Social Change, Mumbai, India \\ ${ }^{3}$ Diabetes Unit, King Edward Memorial Hospital Research Centre, Pune, India \\ ${ }^{4}$ Department of Clinical Pharmacology, Nair Hospital, Mumbai, India \\ ${ }^{5}$ Medical Research Council Human Nutrition Research, Elsie Widdowson Laboratory, Cambridge, UK \\ ${ }^{6}$ Apnalaya, Mumbai, India \\ ${ }^{7}$ Public Health Nutrition, University of Southampton, Southampton, UK \\ (Submitted 28 March 2014 - Final revision received 3 November 2014 - Accepted 6 November 2014 - First published online 13 February 2015)
}

\section{Abstract}

Intakes of micronutrient-rich foods are low among Indian women of reproductive age. We investigated whether consumption of a food-based micronutrient-rich snack increased markers of blood micronutrient concentrations when compared with a control snack. Non-pregnant women ( $n$ 222) aged 14-35 years living in a Mumbai slum were randomised to receive a treatment snack (containing green leafy vegetables, dried fruit and whole milk powder), or a control snack containing foods of low micronutrient content such as wheat flour, potato and tapioca. The snacks were consumed under observation $6 \mathrm{~d}$ per week for 12 weeks, compliance was recorded, and blood was collected at 0 and 12 weeks. Food-frequency data were collected at both time points. Compliance (defined as the proportion of women who consumed $\geq 3$ snacks/week) was $>85 \%$ in both groups. We assessed the effects of group allocation on 12-week nutrient concentrations using ANCOVA models with respective 0-week concentrations, BMI, compliance, standard of living, fruit and green leafy vegetable consumption and use of synthetic nutrients as covariates. The treatment snack significantly increased $\beta$-carotene concentrations (treatment effect: $47 \cdot 1 \mathrm{nmol} / 1,95 \% \mathrm{CI} 6 \cdot 5,87 \cdot 7$ ). There was no effect of group allocation on concentrations of ferritin, retinol, ascorbate, folate or vitamin $\mathrm{B}_{12}$. The present study shows that locally sourced foods can be made into acceptable snacks that may increase serum $\beta$-carotene concentrations among women of reproductive age. However, no increase in circulating concentrations of the other nutrients measured was observed.

Key words: Food-based interventions: India: Micronutrient status

Multiple micronutrient deficiencies are prevalent among women of reproductive age in India ${ }^{(1-3)}$. There are limited data available on the micronutrient status of women living in Indian slums. However, there is evidence that such women are at risk of deficiency. A recent review ${ }^{(4)}$ of studies investigating the intake of micronutrients among women of reproductive age in low- and middle-income countries has found that in Asia, intakes of several micronutrients were frequently below the WHO/FAO Estimated Average Requirement $(E A R)^{(5)}$. The majority of studies reviewed have found that intakes of $\mathrm{Fe}$, folate and vitamin $\mathrm{B}_{12}$ were less than half of the EAR, and between 25 and $77 \%$ of women in Asia had mean intakes of vitamin A and vitamin C below the EAR. No data on carotenoid intakes were reported in the review. The authors suggested that these findings may reflect the cerealbased diets that are commonly consumed in such populations, which tend to be low in fruit, vegetables and animal foods. Low micronutrient intakes have adverse implications for women's risk of anaemia, infectious disease and chronic conditions such as cancer. Optimal offspring health is also dependent on adequate maternal nutrition.

Animal foods are relatively expensive, ${ }^{(6)}$ and a large proportion of the Indian population are vegetarian. Milk, fruit and green leafy vegetables are a good source of micronutrients,

Abbreviations: CRP, C-reactive protein; MMNP, Mumbai Maternal Nutrition Project.

*Corresponding author: S. H. Kehoe, email sk@mrc.soton.ac.uk 
but intakes of these foods are low, with national survey data indicating that in 2005-6 over $50 \%$ of Indian women consumed fruit less than once per week ${ }^{(7)}$. When these data were broken down by region and demographic group, over two-thirds of women living in Mumbai slum areas ( $n$ 803) consumed green leafy vegetables on a daily basis. However, only frequency data were collected in the survey, and several Indian preparations contain very small amounts of green leafy vegetables $(<5 \mathrm{~g})$ such as coriander for seasoning. It is, therefore, possible that the proportion of women who consumed a $100 \mathrm{~g}$ portion daily as recommended in the Indian National Institute of Nutrition guidelines ${ }^{(8)}$ would be considerably smaller than the proportion cited above. A quarter of women reported consuming fruit daily, and approximately $40 \%$ ate fruit less than once per week. Over $15 \%$ of women never consumed milk or curd (yogurt), and 56\% consumed these items less than once per week. Recent quantitative data on dietary intake collected from women living in North Indian slums show that intakes of fruit and vegetables were very low ${ }^{(9,10)}$. Anand et al. ${ }^{(10)}$ found that in slum areas of Haryana state, the mean number of servings per $\mathrm{d}$ of fruit and vegetables was $2 \cdot 2$ for women, with only $5 \cdot 4 \%$ of women consuming five servings per $\mathrm{d}$.

In the present study, we aimed to assess the effects of daily consumption of a food-based intervention in the form of a snack food containing green leafy vegetables, dried fruit and milk powder on blood micronutrient concentrations in nonpregnant, low-income, Indian women of reproductive age.

\section{Methods}

The present study was conducted between October 2009 and March 2010, and was an adjunct to a larger ongoing randomised controlled trial, namely the Mumbai Maternal Nutrition Project $(\mathrm{MMNP})^{(11,12)}$, which was launched in January 2006 (trial registration: ISRCTN 62811278). The MMNP intervention was based on the results of the Pune Maternal Nutrition Study, an observational investigation in a rural area near the city of Pune, which reported that maternal intakes of green leafy vegetables, fruit and milk during pregnancy were positively associated with offspring birth size measurements ${ }^{(13)}$. The MMNP was designed to investigate the effect of maternal consumption of a snack containing green leafy vegetables, fruit and milk for at least 3 months before conception and throughout pregnancy on infant size and mortality. The treatment snack was designed in such a way that in addition to the women's habitual diet, a daily intake of $\geq 75$ th centile of green leafy vegetables (25 g), fruit (10 g) and milk (12 g) as reported in the Pune Maternal Nutrition Study would be achieved.

The present study was a randomised controlled trial of the MMNP intervention model, and was undertaken to examine its effects on micronutrient status among a separate group of non-pregnant women. Baseline status was not assessed in the main MMNP trial in order to minimise blood collection which may have been a deterrent to participation. The present study was conducted approximately 10 miles away from the MMNP study area, and the participants were not enrolled in the MMNP at any time.
The present study was conducted according to the guidelines laid down in the Declaration of Helsinki, and all procedures involving human subjects were approved by the JJ Hospital Ethics Committee, Mumbai.

\section{Participants and settings}

The participants lived in the Shivaji Nagar slum area of Mumbai with inadequate access to safe water and sanitation, poor structural quality of housing and overcrowding. These criteria have been used by the UN to define slums ${ }^{(14)}$. Women were not eligible for the study if they were $<14$ or $>35$ years of age, or reported that they were pregnant or breast-feeding at the time of enrolment. As there is a significant migration rate in the study area, we asked women to enrol only if they intended to remain in the study area for at least 3 months. A power calculation showed that a sample size of eighty-two per group was necessary to demonstrate a difference in the proportion of vitamin A deficiency (defined as serum retinol $<0.7 \mu \mathrm{mol} / \mathrm{l}$ ) of the order of $35 \%$ in the control group $v .10 \%$ in the treatment group at $90 \%$ power and at the 0.05 significance level $^{(15)}$. These estimates were based on data from the WHO Vitamin and Mineral Information System database, whereby the prevalence of vitamin A deficiency ranged from 33 to $35 \%$ in a study of 150 women in urban Calcutta $^{(16)}$. Assuming a dropout rate of $20 \%$, our target sample was 102 in each group.

\section{Procedure}

In October 2009, women living in the Shivaji Nagar area were invited to community meetings at which they were informed about the study by the research team. Written informed consent was obtained from all women who agreed to participate. Those who met the inclusion criteria ( $n$ 222) were enrolled and randomised to the treatment or control arm of the study. Randomisation was stratified by age and BMI to ensure that the groups did not differ by these variables. The treatment and control snacks were outwardly similar, but their contents were different. To achieve a degree of allocation concealment, we created two treatment and two control groups, each with an independent set of recipes. The women were given colour-coded identity cards based on group allocation. There were no differences in the amounts and types of green leafy vegetables, fruit and milk powder in the treatment snacks. A total of four different snacks were produced daily, in an unpredictable pattern. The snacks were packaged in colour-coded bags to match the women's identity cards and ensure that they were correctly distributed. The staff who measured outcomes were blinded to the women's allocation groups. The two treatment groups and the two control groups were merged for analysis.

Baseline data and venous blood samples were collected before the start of supplementation and within $7 \mathrm{~d}$ of enrolment. Demographic data and information on synthetic micronutrient supplement intake in the form of tablets or tonics were collected by means of a questionnaire. A 221-item interviewer-administered FFQ developed for this sample ${ }^{(11)}$ 
was used to collect data on the past week's green leafy vegetable and fruit intake at baseline and at 12 weeks. This was in order to assess any changes in intakes of these foods over the supplementation period. Height was measured to the nearest millimetre using a portable stadiometer (Seca), and weight to the nearest $100 \mathrm{~g}$ using electronic scales (Salter). BMI was expressed as $\mathrm{kg} / \mathrm{m}^{2}$.

During the 12-week supplementation period, women were asked to visit a local community centre $6 \mathrm{~d}$ per week (Monday to Saturday) to receive the snacks and consume them under observation. The centre was $\leq 10$ minutes' walk from the women's homes. Consumption of the snacks was recorded by a project health worker. Compliance with the intervention was defined as consumption of a mean of $\geq 3$ supplements per week over the 12-week period. Follow-up blood samples were collected at 12 weeks after the beginning of supplementation. Women who were found to be moderately or severely anaemic $(\mathrm{Hb}<100 \mathrm{~g} / \mathrm{l})$ at baseline or at 12 weeks were offered Fe tablets. Women who were mildly anaemic (Hb 100-119.9 g/l) were informed of their status, and advised to consult a doctor and consider taking Fe supplements. Anaemic women were eligible to remain in the study.

\section{Intervention}

Women were given one cooked snack per d such as a 'samosa' or 'patty' made from locally available food ingredients. The treatment snacks were designed to provide women with $25 \mathrm{~g}$ fresh green leafy vegetables (e.g. spinach, Colocasia, coriander and fenugreek leaves), $10 \mathrm{~g}$ dried fruit (e.g. Figs, dates and raisins) and $12 \mathrm{~g}$ whole milk powder in addition to their habitual diet. The control snacks contained foods, such as potato, sago or tapioca. Both types of snacks were prepared with binding ingredients, such as wheat or maize flour, to which spices were added. The snacks were cooked by shallow frying in sunflower oil. Several varieties of the treatment ( $n$ 5) and control snacks ( $n$ 7) were developed, to prevent monotony. The average weight of the snacks was approximately $65 \mathrm{~g}$ (treatment) and $36 \mathrm{~g}$ (control). The women were advised to consume the snacks in addition to their habitual diet. One snack per $\mathrm{d}$ was given to the women at a time least likely to interfere with their usual intake, between 15.00 and 18.00 hours, Monday to Saturday.

\section{Nutrient content of the intervention}

Before nutrient analysis, the snacks were frozen in Mumbai and transported on dry ice to the UK, where they were analysed at Eclipse Laboratories (Cambridge, UK) for micronutrient content. Over $75 \%$ of the recipes were analysed and mean micronutrient content was derived (Table 1).

To assess mineral content, samples were dried and ashed at $550^{\circ} \mathrm{C}$ for $16 \mathrm{~h}$, then dissolved in $5 \mathrm{M}-\mathrm{HCl}$, and scandium (internal standard)/caesium chloride solution was added. After filtration and dilution to known volumes with water, the concentration of each mineral was determined by 'Liberty series II' inductively coupled plasma atomic emission spectrometry.

Ascorbic acid was extracted from the sample using metaphosphoric acid and EDTA. Ascorbic acid was then enzymatically oxidised to dehydroascorbic acid, which was condensed with $O$-phenylenediamine to give the fluorescent quinoxaline derivative. The latter was separated from interfering compounds by reverse-phase HPLC with fluorometric detection.

$\beta$-Carotene analysis was conducted away from natural light using amber glassware. The sample was saponified for $30 \mathrm{~min}$ at $95^{\circ} \mathrm{C}$ with ethanolic potassium hydroxide, and carotene was extracted with hexane. Hexane was evaporated to dryness and carotene dissolved in the mobile phase and quantified by HPLC with UV detection, against a calibration standard of known concentration.

Folates were extracted from the samples using $0 \cdot 1 \mathrm{M}$-potassium phosphate buffer and by heating for $15 \mathrm{~min}$ at $100^{\circ} \mathrm{C}$. The filtrate was diluted to a suitable level and treated with deconjugase enzyme. L-Ascorbic acid was also added to prevent oxidation. This complex was incubated at $37^{\circ} \mathrm{C}$ for $4 \mathrm{~h}$. Sample extracts were diluted to values within the calibrated range. Folic acid casei media were added to the diluted samples, which were then covered with aluminium foil and sterilised at $121^{\circ} \mathrm{C}$ in an autoclave. The assay was inoculated

Table 1. Mean nutrient composition and mean percentage contribution to nutrient requirements of the treatment and control snacks (Mean values and standard deviations)

\begin{tabular}{|c|c|c|c|c|c|c|c|c|c|c|c|c|}
\hline & \multicolumn{12}{|c|}{ Nutrient content } \\
\hline & \multicolumn{2}{|c|}{ Energy† (MJ) } & \multicolumn{2}{|c|}{$\mathrm{Fe}(\mathrm{mg})$} & \multicolumn{2}{|c|}{$\mathrm{RE}(\mu \mathrm{g})$} & \multicolumn{2}{|c|}{ Vitamin C (mg) } & \multicolumn{2}{|c|}{ Folate $\neq(\mu \mathrm{g})$} & \multicolumn{2}{|c|}{ Vitamin $B_{12}(\mu \mathrm{g})$} \\
\hline & Mean & SD & Mean & SD & Mean & SD & Mean & SD & Mean & SD & Mean & SD \\
\hline \multicolumn{13}{|l|}{ Treatment snacks (mean weight $65 \mathrm{~g}$ ) } \\
\hline Nutrient content per serving & 0.61 & 0.07 & 3.9 & 1.3 & 141 & 85 & $2 \cdot 1$ & 3.0 & 67.5 & $30 \cdot 6$ & 0.31 & 0.13 \\
\hline Percentage of RNI§ per serving & & & \multicolumn{2}{|c|}{20} & 18 & & \multicolumn{2}{|l|}{4} & \multicolumn{2}{|c|}{11} & \multicolumn{2}{|c|}{12} \\
\hline \multicolumn{13}{|l|}{ Control snacks (mean weight $36 \mathrm{~g}$ ) } \\
\hline Nutrient content per serving & 0.37 & 0.05 & 0.9 & 0.26 & 2 & 1 & 0.0 & 0.0 & $6 \cdot 1$ & 4.6 & 0.18 & 0.25 \\
\hline Percentage of RNI§ per serving & & & \multicolumn{2}{|c|}{5} & \multicolumn{2}{|c|}{$<1$} & \multicolumn{2}{|c|}{$<1$} & \multicolumn{2}{|c|}{1} & \multicolumn{2}{|c|}{7} \\
\hline
\end{tabular}

$\mathrm{RE}$, retinol equivalents; RNI, reference nutrient intake.

${ }^{*}$ Weighted average based on the number of days that the snacks were distributed over the study period.

$\dagger$ Energy content was calculated from the Indian Food Composition Tables ${ }^{(17)}$.

$\ddagger$ Total folate.

$\S \mathrm{FAO} / \mathrm{WHO}-$ recommended reference nutrient intake during the first trimester of pregnancy ${ }^{(5)}$. 
with Lactobacillus rhamnosus and incubated overnight at $37^{\circ} \mathrm{C}$. The concentration of folate in the sample was measured spectrophotometrically.

The energy content of the snacks was calculated using the values for raw ingredients from Indian Food Composition Tables ${ }^{(17)}$.

\section{Biochemical measurements}

Within $1 \mathrm{~h}$ from collection, venous blood was centrifuged at $20^{\circ} \mathrm{C}$ for $10 \mathrm{~min}$ (REMY). Depending on the assay, plasma or serum was pipetted into vials and kept on dry ice for up to $8 \mathrm{~h}$ before being transported to a $-80^{\circ} \mathrm{C}$ freezer for storage until analysis. For vitamin $\mathrm{C}$ analysis, $0.3 \mathrm{ml}$ plasma was added to $0.3 \mathrm{ml}$ of $10 \%$ metaphosphoric acid and stored at $-80^{\circ} \mathrm{C}$ until analysis.

A quantitative test kit based on solid-phase ELISA was used to measure serum ferritin concentrations (ELISA; Diagnostic Automation, Inc.). The system used one anti-ferritin antibody for solid-phase (microtitre wells) immobilisation and another mouse monoclonal anti-ferritin antibody in the antibodyenzyme (horseradish peroxidase) conjugate solution. The test sample was allowed to react simultaneously with the antibodies, resulting in the ferritin molecules being sandwiched between the solid phase and enzyme-linked antibodies. After incubation at room temperature for $60 \mathrm{~min}$, the wells were washed with water to remove unbound antibodies. A solution of tetramethylbenzidine was added and incubated for $20 \mathrm{~min}, 2 \mathrm{M}-\mathrm{HCl}$ was added, and ferritin concentration was measured spectrophotometrically at $450 \mathrm{~nm}$.

For the measurement of $\beta$-carotene, thawed subsamples of plasma were extracted with $n$-hexane (containing butylated hydroxytoluene) in the presence of aqueous SDS and absolute ethanol (the latter containing $\alpha$-tocopherol acetate (internal standard)). The upper organic phase was evaporated to dryness under vacuum and then redissolved in $250 \mu \mathrm{l}$ of the mobile phase. Aliquots $(50 \mu \mathrm{l})$ were then injected onto a $3 \mu \mathrm{m}$ YMC-Pack Pro $\mathrm{C}_{18}$ column. The mobile phase was acetonitrile (44\%), methanol (44\%) and dichloromethane (12\%), by volume. For each sample, $\beta$-carotene was measured at $450 \mathrm{~nm}$ using an Empower 2-controlled HPLC system (Waters Ltd), with a photodiode array detector. Simultaneously, the internal standard was measured at $284 \mathrm{~nm}$, and the ratio of the responses calculated (response factor). $\beta$-Carotene calibration lines were obtained from semi-pure, commercially available $\beta$-carotene. The concentration of the calibrants was determined from their absolute optical densities and known extinction coefficient. These were then corrected to $100 \%$ purity, by means of their HPLC purity profiles ${ }^{(18)}$.

Retinol levels were determined using a standard HPLC kit (Recipe Chemicals + Instruments, GmbH). Sample preparation involved two steps: (1) extraction and (2) stabilisation. First, $150 \mu \mathrm{l}$ of calibrator/control/serum and $150 \mu \mathrm{l}$ of precipitant $\mathrm{P}$ (containing internal standard) were mixed in a sample preparation vial, and then centrifuged at $10000 \boldsymbol{g}$ for $5 \mathrm{~min}$. Second, stabilisation was performed by mixing $100 \mu \mathrm{l}$ of the centrifuged supernatant and $100 \mu \mathrm{l}$ cooled stabilising reagent $\mathrm{S}$ followed by centrifugation at $10000 \mathrm{~g}$ for $5 \mathrm{~min}$. A $50 \mu \mathrm{l}$ supernatant was injected into the Thermo Spectra Isocratic HPLC System with a UV detector (Thermo Fisher Scientific Private Limited). Injection volume was $50 \mu \mathrm{l}$ with a flow rate of $1.5 \mathrm{ml} / \mathrm{min}$. The initial wavelength was $325 \mathrm{~nm}$ and after $3.5 \mathrm{~min}$, the wavelength was altered to $295 \mathrm{~nm}$. The column temperature was maintained at $30^{\circ} \mathrm{C}$. The retention time for retinol was within $2 \mathrm{~min}$ and for the internal standard was $5 \mathrm{~min}$. The limit of detection was $10 \mathrm{ng} / \mathrm{ml}$, and the limit of quantification $20 \mathrm{ng} / \mathrm{ml}$. For validation of data, ClinChek ${ }^{\circledR}$ Serum Control Levels I and II (Recipe Chemicals + Instruments, GmbH) were used along with each batch of samples ${ }^{(19)}$.

Ascorbic acid concentrations were assayed using an in-house method based on the procedure described by Vuilleumier \& $\mathrm{Keck}^{(20)}$, adapted for microplates. Ascorbic acid in the metaphosphoric acid-stabilised plasma sample was converted into dehydroascorbic acid by ascorbate oxidase (Sigma). The resulting dehydroascorbate was coupled with O-phenylenediamine to give a fluorescent quinoxaline. The formation of this quinoxaline was linearly related to the amount of vitamin $\mathrm{C}$ in the sample, over the range of $0-10 \mu \mathrm{g} / \mathrm{ml}(0-5 \mu \mathrm{m})$. Fluorescence was measured using a BMG LABTECH FLUOstar OPTIMA plate reader. The accuracy of the fluorometric assay procedure used was ascertained by participation in the external quality assessment programme, provided by the National Institute of Science and Technology, USA, which validates results against Standard Reference Preparation no. 970 .

Plasma folate concentrations were measured by microbiological assay using a chloramphenicol-resistant strain of Lactobacillus casei, and using Victor-2 (PerkinElmer Life Science $)^{(21,22)}$. Plasma vitamin $\mathrm{B}_{12}$ was measured by microbiological assay using a colistin sulphate-resistant strain of Lactobacillus leichmannii ${ }^{(23,24)}$.

Plasma C-reactive protein (CRP) was measured to assess levels of acute-phase reactants to interpret the results of serum ferritin and retinol analysis. CRP was assayed using a high-sensitivity ELISA kit (United Biotech), and using Victor-2 (PerkinElmer Life Science). The CV for the assay was $<11 \%$. The cut-off value for active inflammation was $5000 \mathrm{ng} / \mathrm{ml}^{(25)}$.

\section{Statistical analysis}

Where data were normally distributed, means and standard deviations are presented, and elsewhere medians and interquartile ranges (IQR) are given. BMI was log-transformed for regression analysis. Independent $t$ tests and $\chi^{2}$ tests were used to assess the differences in baseline characteristics between intervention groups. Paired $t$ tests were used to assess within-group changes in fruit and green leafy vegetable consumption over the study period. Independent $t$ tests were used to assess the differences between groups with respect to the change in fruit and green leafy vegetable consumption at baseline and at 12 weeks. ANCOVA models for each nutrient were used to assess treatment effects with 12-week concentrations as dependent variables and respective baseline concentration, age, BMI, standard of living index, compliance 
with supplementation, use of nutritional supplements, 12-week fruit intake frequency and 12-week green leafy vegetable intake frequency as covariates.

In the case of ferritin and retinol, women with CRP concentrations $>5000 \mathrm{ng} / \mathrm{ml}$ were excluded from the analyses, ${ }^{(26,27)}$ and the models were adjusted for plasma CRP concentrations. All statistical analyses were performed using the SPSS software package version 19 (SPSS, Inc.).

\section{Results}

\section{Participant characteristics}

Table 2 presents the anthropometric and demographic characteristics of the women enrolled in the trial, as well as the proportion of women who were nutrient deficient at baseline. A large proportion of the women in the study were underweight and chronically energy deficient based on BMI. The majority of women were of Muslim faith. Over three-quarters of the women had completed at least secondary level participants and percentages) education. A third of them were in paid employment at the time of the study. Our data indicate that the majority of the women were Fe deficient, about one-fifth were retinol deficient (using the $1.047 \mu \mathrm{mol} / \mathrm{l}$ cut off) and two-fifths were vitamin $\mathrm{C}$ deficient. Fewer than $10 \%$ had low folate or vitamin $\mathrm{B}_{12}$ status. Almost two-thirds were mildly anaemic, and very few severely anaemic. There were no significant differences in any of the baseline characteristics between the control and treatment groups.

During the study, thirty women reported taking synthetic micronutrient supplements. Of these, eighteen took multiple micronutrient supplements, eight took $\mathrm{Fe}$ and folate, and four took Fe. The median monthly family income was 4000 (IQR 3000-5000) rupees equivalent to approximately USD88 (IQR USD66-110), and the median family size was six (IQR 5-7) persons. Approximately $15 \%$ of the women lived in 'katcha'-style housing made from plastic sheeting, sticks and textiles. The remainder had 'semi-pucca' or 'pucca' walls and floors, made with cement and corrugated metal roofs. Most women $(86 \%)$ used a shared pit toilet.

Table 2. Baseline characteristics of the women by intervention group (all 222 women enrolled) (Mean values and standard deviations; medians and interquartile ranges (IQR); number of

\begin{tabular}{|c|c|c|c|c|c|}
\hline & \multicolumn{2}{|c|}{ Control ( $n$ 114) } & \multicolumn{2}{|c|}{ Treatment $(n$ 108) } & \multirow[b]{2}{*}{$P^{\star}$} \\
\hline & Median & IQR & Median & IQR & \\
\hline Age (years) & 20 & $17-25$ & 21 & $17-27$ & 0.999 \\
\hline Weight (kg) & 41.6 & $35 \cdot 7-46 \cdot 8$ & $40 \cdot 0$ & $36 \cdot 7-46 \cdot 8$ & 0.477 \\
\hline Height $(\mathrm{cm})$ & & & & & 0.750 \\
\hline Mean & & $9 \cdot 6$ & & $9 \cdot 5$ & \\
\hline SD & & 7 & & 6 & \\
\hline \multirow[t]{2}{*}{$\mathrm{BMI}\left(\mathrm{kg} / \mathrm{m}^{2}\right)$} & $18 \cdot 6$ & $16 \cdot 3-20 \cdot 1$ & $18 \cdot 4$ & $16 \cdot 4-20 \cdot 5$ & 0.626 \\
\hline & $n$ & $\%$ & $n$ & $\%$ & \\
\hline \multicolumn{5}{|l|}{ Religion } & 0.593 \\
\hline Hindu & 20 & 17.5 & 14 & 13.0 & \\
\hline Muslim & 90 & 78.9 & 91 & $84 \cdot 3$ & \\
\hline Other & 4 & 3.5 & 3 & $2 \cdot 8$ & \\
\hline \multicolumn{5}{|l|}{ Education } & 0.181 \\
\hline Primary or less & 27 & $23 \cdot 7$ & 19 & $17 \cdot 6$ & \\
\hline Secondary & 80 & $70 \cdot 2$ & 78 & $72 \cdot 2$ & \\
\hline Graduate & 7 & $6 \cdot 2$ & 5 & 4.6 & \\
\hline \multicolumn{5}{|l|}{ Occupation } & 0.587 \\
\hline Semi-skilled/unskilled & 27 & $23 \cdot 7$ & 25 & $23 \cdot 1$ & \\
\hline Skilled/self-employed & 13 & 11.4 & 6 & $5 \cdot 6$ & \\
\hline Professional & 4 & 3.5 & 2 & 1.9 & \\
\hline Not working & 70 & 61.4 & 75 & $69 \cdot 4$ & \\
\hline \multicolumn{6}{|l|}{ Deficiency status } \\
\hline Ferritin $(<15 \mathrm{ng} / \mathrm{ml}) \dagger$ & 100 & $87 \cdot 7$ & 85 & $78 \cdot 7$ & 0.258 \\
\hline Ferritin $(<15 \mathrm{ng} / \mathrm{ml}) \ddagger$ & 61 & 88.4 & 55 & 84.6 & 0.520 \\
\hline Retinol $(<1.05 \mu \mathrm{mol} / \mathrm{l})$ & 20 & $18 \cdot 5$ & 24 & $22 \cdot 2$ & 0.360 \\
\hline Retinol $(<1.05 \mu \mathrm{mol} / \mathrm{l}) \ddagger$ & 17 & $19 \cdot 1$ & 16 & $19 \cdot 0$ & 0.972 \\
\hline Ascorbate $(<11 \mu \mathrm{mol} / \mathrm{l})$ & 46 & $40 \cdot 4$ & 38 & $35 \cdot 1$ & 0.385 \\
\hline Folate $(<6.8 \mathrm{nmol} / \mathrm{l})$ & 9 & 7.9 & 9 & $8 \cdot 3$ & 0.805 \\
\hline Vitamin $\mathrm{B}_{12}(<150 \mathrm{pmol} / \mathrm{l})$ & 3 & $2 \cdot 6$ & 2 & 1.9 & 0.250 \\
\hline $\mathrm{Hb}(<120 \mathrm{~g} / \mathrm{l})$ & 69 & 60.5 & 71 & $65 \cdot 7$ & 0.844 \\
\hline $\mathrm{Hb}(<80 \mathrm{~g} / \mathrm{l})$ & 3 & $2 \cdot 6$ & 1 & 0.9 & 0.346 \\
\hline
\end{tabular}




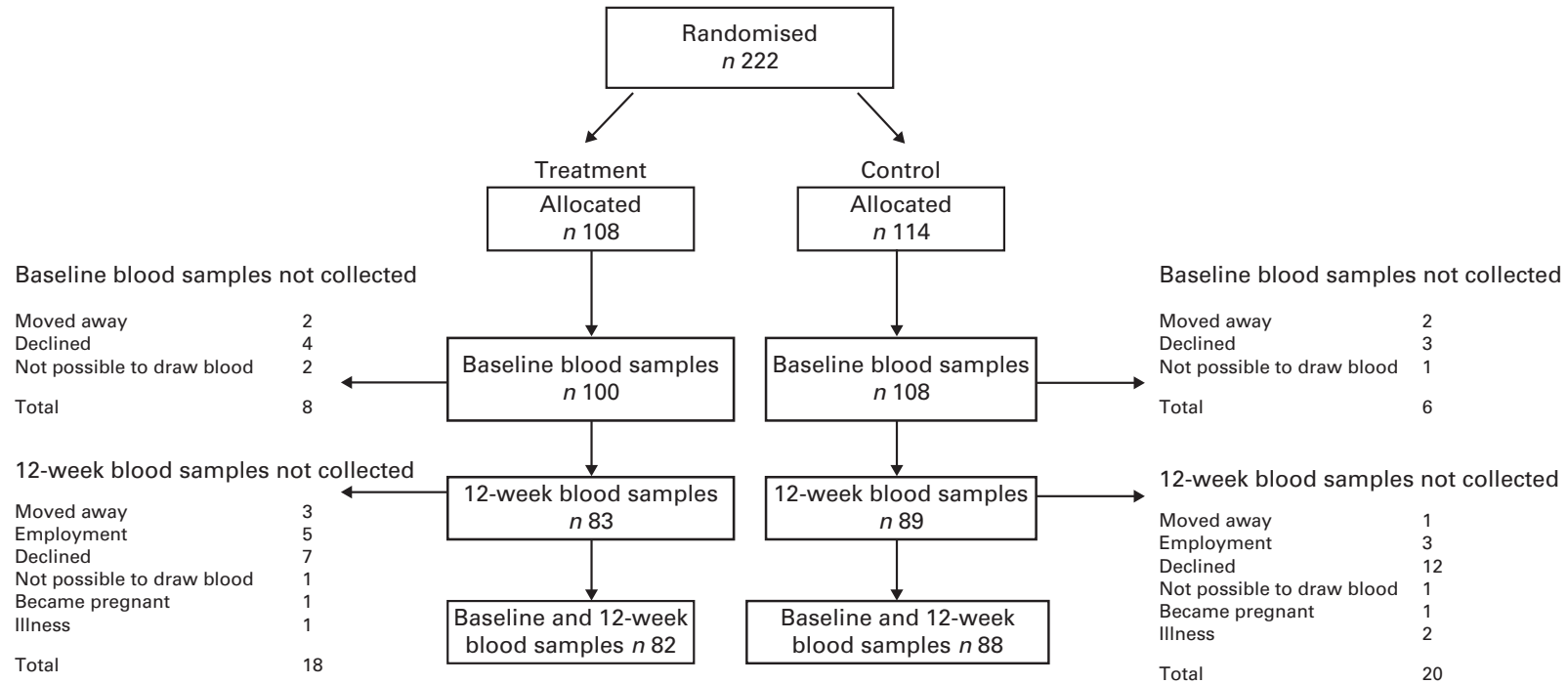

Fig. 1. Flow chart of the study participants.

\section{Participant flow}

Of the 222 women randomised, a total of 208 women with baseline blood measurements started receiving the intervention (Fig. 1). Of these, blood was collected at 12 weeks from 172 women and blood samples were available at both time points for 170 women. Compliance (defined as the proportion of women who consumed $\geq 3$ snacks per week) was $85 \%$ in the treatment and $90 \%$ in the control groups. The median weekly snack consumption of those in the final analysis was $5 \cdot 0$ (IQR $4 \cdot 4-5 \cdot 5)$ in the control group and 4.9 (IQR $3 \cdot 8-5 \cdot 3$ ) in the treatment group.

\section{Dietary intake}

Habitual fruit intake increased in both groups: from a mean of $2 \cdot 1$ (SD 3.1) to 3.9 (SD 5.5) servings per week in the control group $(P=0 \cdot 001)$, and from $2 \cdot 0(\mathrm{sD} 3 \cdot 1)$ to $3 \cdot 8$ (SD $5 \cdot 1)$ servings per week in the treatment group $(P=0.003)$. There was no difference between the groups in terms of the change in fruit intake $(P=0.987)$. Habitual intakes of green leafy vegetables in the control group increased by a mean of 0.8 (SD $2 \cdot 2$ ) servings per week from $1 \cdot 3$ (SD $1 \cdot 4$ ) to $2 \cdot 1$ (SD $2 \cdot 0$ ) servings per week $(P<0 \cdot 001)$. There was no difference between 0 -week and 12 -week green leafy vegetable intakes in the treatment group. Mean intakes were 1.4 (SD 1.7) servings per week at baseline and $1.7(\mathrm{SD} 2.6)$ at 12 weeks $(P=0.420)$. The difference between the groups in terms of the change in intakes over the study period did not reach any statistical significance $(P=0 \cdot 144 ;$ data not shown).

\section{Blood micronutrient concentrations}

There was no effect of the treatment on serum ferritin concentrations (Table 3). When we excluded the women whose CRP levels were $>5000 \mathrm{ng} / \mathrm{ml}$ ( $n$ 28) and reran the analysis, there was little difference in the median ferritin concentrations observed. The data indicated a statistically significant treatment effect on $\beta$-carotene concentrations of $47 \mathrm{nmol} / \mathrm{l}$. This was approximately $12 \%$ of the baseline concentrations. The CV of the $\beta$-carotene assay was approximately $15 \%$. No effect of group allocation was observed for retinol, ascorbate, folate or vitamin $\mathrm{B}_{12}$.

\section{Discussion}

The present study aimed to assess the effects of consumption of a micronutrient-rich food-based intervention on the nutritional status of low-income group Indian women. We found that consumption of the treatment snacks for 12 weeks was associated with a small increase in $\beta$-carotene concentrations relative to the control group. The mean difference in the change between groups was $47 \mathrm{nmol} / 1$, which was just over $12 \%$ of baseline $\beta$-carotene values. The $\mathrm{CV}$ of the assay was of a similar magnitude, but due to the randomised design, it could be reasonably expected that the measurement error would not differ between the groups. There was no significant effect of the intervention allocation on the change in the concentrations of any of the other micronutrients studied.

In the UK, the effect of adding $85 \mathrm{~g} / \mathrm{d}$ of raw watercress to the usual diet for 8 weeks was an increase in $\beta$-carotene levels by a mean of $100 \mathrm{nmol} / \mathrm{l}^{(28)}$. Considering the quantity of green leafy vegetables in the intervention in the present study, it would appear that the effect size is consistent with this finding.

The treatment effect in the present study was considerably smaller than that in studies where alterations to the entire dietary pattern such as increasing daily intake of fruit and vegetables from two to ten portions per d were implemented. Such interventions have achieved up to a fivefold increase in $\beta$-carotene concentrations ${ }^{(29-31)}$. It is questionable how sustainable such changes to diets would be in the Indian slum population.

There are inconsistent effects of vitamin A-rich foods on nutritional status among populations in Asia and Africa. Among Bangladeshi men, vitamin A pool size was increased 
following a spinach intervention ${ }^{(32)}$. Among Kenyan children, it has been reported that dehydrated amaranth and cowpea leaves increased serum $\beta$-carotene and retinol concentrations ${ }^{(33)}$. However, a recent study in Bangladesh assessed the effect of the consumption of bio-fortified orange-fleshed sweet potato on vitamin A pool size among vitamin A-deficient women living in a low-income area of Dhaka city ${ }^{(34)}$. The potato intervention increased circulating $\beta$-carotene concentrations, but there was no effect on vitamin A stores. This finding indicated that the conversion of $\beta$-carotene to vitamin $\mathrm{A}$ was limited in this population. The authors concluded that protein deficiencies among these women may have led to reduced synthesis of BCMO1 ( $\beta$-carotene $15,15^{\prime}$-monooxygenase 1 ), which, in turn, would reduce the conversion of $\beta$-carotene to retinol.

Over three-quarters of the women in the present study had retinol and folate concentrations above the cut-offs for deficiency. Therefore, it is possible that the majority of the women in this sample were not deficient in these nutrients. In addition, the duration of the supplementation period may have been too short for retinol status to be altered. It was necessary for us to achieve a balance between resources, compliance with the protocol and observing an effect of the supplement on micronutrient status. Furthermore, in the case of vitamin C, the content in the snacks was low, probably due to the cooking process. There was some experimentation with different methods of cooking, and preparing the snacks to preserve vitamin C content, but the most acceptable method to the women was shallow frying, and this method was used, as it was important to achieve adherence to the protocol.

A study on a small group of healthy young females in Pune, West India, compared the short-term effects of consumption of a green leafy vegetable meal with a standard meal without green leafy vegetables ${ }^{(35)}$. No difference in plasma $\beta$-carotene or vitamin $\mathrm{C}$ concentrations between the two groups was observed $4 \mathrm{~h}$ after the meal. However, after a 3-week intervention period constituting daily supplementation with $100 \mathrm{~g}$ cooked green leafy vegetables and $10 \mathrm{~g}$ oil, there was a significant increase in plasma concentrations of both nutrients. The authors concluded that an intake of $100 \mathrm{~g}$ green leafy vegetables per d plus $10 \mathrm{~g}$ oil could be an effective strategy for improving micronutrient status of young Indian women. The treatment snacks in the present study contained approximately $25 \mathrm{~g}$ green leafy vegetables per serving; it is possible that there is a 'threshold nutrient intake' above which a change in nutrient concentration would be observed. For example, consumption of four snacks per $\mathrm{d}$ may have led to significant increases in the concentrations of the other nutrients, and a more marked increase in $\beta$-carotene concentrations in the treatment group.

We assessed the possible impact of inflammation on ferritin status using CRP concentrations; however, this did not alter the lack of association with treatment allocation. The snacks contained almost $4 \mathrm{mg}$ Fe per serving. However, it is likely that there was insufficient bioavailable $\mathrm{Fe}$ in the snacks due to the inhibition of Fe uptake, attributable, in turn, to frequent consumption of phytate-containing cereal-based foods, tea containing polyphenols, milk protein and polyphenols in green leafy vegetables in the snacks themselves. 
The present study had several strengths and limitations. Before the study, there was a paucity of data on the effect of long-term food-based interventions on the nutritional status of women in low-income settings. We used a randomised controlled design, but as with many food-based intervention studies, it was not possible to blind the intervention. It is unlikely that this would have influenced the findings, given that the outcomes were objectively assessed, and the group allocations were not known to the laboratory staff who made the nutrient concentration measurements. A strength of the study is that all of the participants started and completed the intervention at the same time, thus creating an internal control for seasonal changes in food intakes.

The sample size calculation was based on serum retinol concentrations, as this was the nutrient for which data were available for the Indian population. Based on previously published data, we had perhaps overestimated the proportion of women who would be retinol deficient, and it is possible that the study was underpowered for other nutrients, for which no status data of this sample were available. It would have been of interest to have measured the change in $\mathrm{Zn}$ status, as green leafy vegetables are a source of this mineral, but circulating $\mathrm{Zn}$ concentrations are tightly regulated, and this would have required a different methodology which was not feasible within the present study.

It is possible that the women might have changed their habitual diet on account of being enrolled in the study; it is also conceivable that women in the treatment group reduced their consumption of green leafy vegetables because they were aware of the snack intervention. Women in the control group may have done the opposite, thus negating the effect of the snacks on micronutrient status. Our data suggest that this did happen to a certain extent in the present study, and the effect size that we have shown in relation to $\beta$-carotene concentrations may be an underestimation. A similar phenomenon was observed in the UK school fruit scheme, whereby children who received fruit at school were given less fruit at home, and so an intervention designed to increase children's intake of fruit by one portion per $\mathrm{d}$ resulted in only a 0.5 portion per $\mathrm{d}$ increase $^{(36)}$, and this was perhaps due to the parents' assumption that children required less fruit at home in the context of the school fruit scheme ${ }^{(37)}$. This phenomenon poses a crucial challenge to the design of food-based interventions.

In conclusion, the present study indicated that small changes in intakes of fruit, green leafy vegetables and milk in a food-based snack intervention might have led to increased levels of circulating $\beta$-carotene concentrations in the sample chosen.

\section{Acknowledgements}

The authors thank the study participants and their families for their time and commitment to the study; staff from Apnalaya and Centre for Study of Social Change, Mumbai, for their contribution to the fieldwork; Diabetes Unit, King Edward Memorial Hospital, Pune, Medical Research Council Human Nutrition Research Unit, Cambridge, ALS Food and Pharmaceutical and Nair Hospital, Mumbai, for laboratory analyses and advice on assay preparation.
The present study was funded by the Wellcome Trust, ICICI Bank and the Medical Research Council Lifecourse Epidemiology Unit.

The authors' contributions are as follows: S. H. K. contributed to the conception and design of the study, data collection and analysis, and wrote the manuscript; C. H. D. F. and B. M. M. contributed to the conception and design of the study and reviewed/edited the manuscript; H. C. and D. T. contributed to the design of the study, data collection and reviewed/ edited the manuscript; S. A. S., N. B., M. G. and R. D. P. contributed to the design of the study and reviewed/edited the manuscript; D. B., R. P. M., F. P. and S. Y. contributed to the data collection and biochemical analysis and reviewed/ edited the manuscript.

None of the authors has any conflict of interest.

\section{References}

1. World Health Organization (2008) Worldwide Prevalence of Anaemia 1993-2005 WHO Global Database on Anaemia. Geneva: WHO.

2. Pathak P, Kapil U, Yajnik CS, et al. (2007) Iron, folate, and vitamin B12 stores among pregnant women in a rural area of Haryana State, India. Food Nutr Bull 28, 435-438.

3. Black RE, Allen LH, Bhutta ZA, et al. (2008) Maternal and child undernutrition: global and regional exposures and health consequences. Lancet 371, 243-260.

4. Torheim LE, Ferguson EL, Penrose K, et al. (2010) Women in resource-poor settings are at risk of inadequate intakes of multiple micronutrients. J Nutr 140, 2051S-2058S.

5. Food and Agriculture Organization (2004) Vitamin and Mineral Requirements in Human Nutrition. Joint FAO/WHO Expert Consultation on Human Vitamin and Mineral Requirements, 2 ed. Geneva: World Health Organization.

6. Maillot M, Darmon N, Darmon M, et al. (2007) Nutrientdense food groups have high energy costs: an econometric approach to nutrient profiling. J Nutr 137, 1815-1820.

7. International Institute for Population Sciences (2007) National Family Health Survey (NFHS-3) 2005-O6 Volume 1. Mumbai: International Institute for Population Sciences.

8. National Institute of Nutrition, Indian Council of Medical Research (2010) Dietary Guidelines for Indians, 2 ed. Hyderabad: National Institute of Nutrition.

9. Yadav K \& Krishnan A (2008) Changing patterns of diet, physical activity and obesity among urban, rural and slum populations in north India. Obes Rev 9, 400-408.

10. Anand K, Shah B, Yadav K, et al. (2007) Are the urban poor vulnerable to non-communicable diseases? A survey of risk factors for non-communicable diseases in urban slums of Faridabad. Natl Med J India 20, 115-120.

11. Shivshankaran D, Gurmurthy S, Kehoe SH, et al. (2011) Developing micronutrient-rich snacks for pre-conception and antenatal health: the Mumbai Maternal Nutrition Project. In Combating Micronutrient Deficiencies: Food-based Approaches, pp. 214-223 [B Thompson and L Amoroso, editors]. Rome: Food and Agriculture Organization of the United Nations.

12. Potdar RD, Sahariah SA, Gandhi M, et al. (2014) Improving women's diet quality preconceptionally and during gestation: effects on birth weight and prevalence of low birth weight - a randomized controlled efficacy trial in India (Mumbai Maternal Nutrition Project). Am J Clin Nutr 100, 1257-1268. 
13. Rao S, Yajnik CS, Kanade A, et al. (2001) Intake of micronutrient-rich foods in rural Indian mothers is associated with the size of their babies at birth: Pune Maternal Nutrition Study. J Nutr 131, 1217-1224.

14. United Nations Human Settlements Programme (2003) The Challenge of Slums, Global Report on Human Settlements 2003. London: Routledge.

15. Lwanga SK \& Lemeshow S (1991) Sample Size Determination in Health Studies: A Practical Manual. Geneva: WHO.

16. Basu S, Sengupta B \& Paladhai PK (2003) Single megadose vitamin A supplementation of Indian mothers and morbidity in breastfed young infants. Postgrad Med J 79, 397-402.

17. Gopalan C, Sastri R \& Balasubramanian SC (1989) Nutritive Value of Indian Foods. Hyderabad, India: National Institute of Nutrition.

18. Thurnham DI, Smith E \& Flora PS (1988) Concurrent liquidchromatographic assay of retinol, $\alpha$-tocopherol, $\beta$-carotene, $\alpha$-carotene, lycopene, and $\beta$-cryptoxanthin in plasma, with tocopherol acetate as internal standard. Clin Chem $\mathbf{3 4}$, $377-381$.

19. Siddiqui FQ, Malik F \& Fazli FR (1995) Determination of serum retinol by reversed-phase high-performance liquid chromatography. J Chromatogr B Biomed Appl 666, 342-346.

20. Vuilleumier JP \& Keck E (1989) Fluorometric assay of vitamin C in biological materials using a centrifugal analyser with fluorescence attachment. J Micronutrient Anal 5, 25-34.

21. Horne DW \& Patterson D (1988) Lactobacillus casei microbiological assay of folic acid derivatives in 96-well microtiter plates. Clin Chem 34, 2357-2359.

22. Tamura T, Freeberg LE \& Cornwell PE (1990) Inhibition of EDTA of growth of Lactobacillus casei in the folate microbiological assay and its reversal by added manganese or iron. Clin Chem 36, 1993.

23. Kelleher BP, Walshe KG, Scott JM, et al. (1987) Microbiological assay for vitamin B12 with use of a colistinsulfate-resistant organism. Clin Chem 33, 52-54.

24. Kelleher BP \& Broin SD (1991) Microbiological assay for vitamin B12 performed in 96-well microtitre plates. J Clin Pathol 44, 592-595.

25. Woodruff BA, Blanck HM, Slutsker L, et al. (2006) Anaemia, iron status and vitamin A deficiency among adolescent refugees in Kenya and Nepal. Public Health Nutr 9, 26-34.

26. Thurnham DI, McCabe GP, Northrop-Clewes CA, et al. (2003) Effects of subclinical infection on plasma retinol concentrations and assessment of prevalence of vitamin A deficiency: meta-analysis. Lancet 362, 2052-2058.
27. Thurnham DI, McCabe LD, Haldar S, et al. (2010) Adjusting plasma ferritin concentrations to remove the effects of subclinical inflammation in the assessment of iron deficiency: a meta-analysis. Am J Clin Nutr 92, 546-555.

28. Gill CI, Haldar S, Boyd LA, et al. (2007) Watercress supplementation in diet reduces lymphocyte DNA damage and alters blood antioxidant status in healthy adults. Am J Clin Nutr 85, 504-510.

29. Maramag CC, Ribaya-Mercado JD, Rayco-Solon $\mathrm{P}$, et al. (2010) Influence of carotene-rich vegetable meals on the prevalence of anaemia and iron deficiency in Filipino schoolchildren. Eur J Clin Nutr 64, 468-474.

30. Chopra M, O'Neill ME, Keogh N, et al. (2000) Influence of increased fruit and vegetable intake on plasma and lipoprotein carotenoids and LDL oxidation in smokers and nonsmokers. Clin Chem 46, 1818-1829.

31. Paterson E, Gordon MH, Niwat C, et al. (2006) Supplementation with fruit and vegetable soups and beverages increases plasma carotenoid concentrations but does not alter markers of oxidative stress or cardiovascular risk factors. J Nutr 136, 2849-2855.

32. Haskell MJ, Jamil KM, Hassan F, et al. (2004) Daily consumption of Indian spinach (Basella alba) or sweet potatoes has a positive effect on total-body vitamin A stores in Bangladeshi men. Am J Clin Nutr 80, 705-714.

33. Nawiri MP, Nyambaka H \& Murungi JI (2013) Sun-dried cowpeas and amaranth leaves recipe improves $\beta$-carotene and retinol levels in serum and hemoglobin concentration among preschool children. Eur J Nutr 52, 583-589.

34. Jamil KM, Brown KH, Jamil M, et al. (2012) Daily consumption of orange-fleshed sweet potato for 60 days increased plasma $\beta$-carotene concentration but did not increase total body vitamin A pool size in Bangladeshi women. J Nutr 142, 1896-1902.

35. Agte V, Jahagirdar M \& Chiplonkar S (2006) GLV supplements increased plasma $\beta$-carotene, vitamin $C$, zinc and hemoglobin in young healthy adults. Eur J Nutr 45, 29-36.

36. Ransley JK, Greenwood DC, Cade JE, et al. (2007) Does the school fruit and vegetable scheme improve children's diet? A non-randomised controlled trial. J Epidemiol Community Health 61, 699-703.

37. Schagen S, Blenkinsop S, Schagen I, et al. (2005) Evaluation of the School Fruit and Vegetable Pilot Scheme. London: Big Lottery Fund. 